\title{
Neutron resonance analysis for nuclear safeguards and security applications
}

\author{
Carlos Paradela ${ }^{1, \text { a }}$, Jan Heyse ${ }^{1}$, Stefan Kopecky ${ }^{1}$, Peter Schillebeeckx ${ }^{1}$, Hideo Harada ${ }^{2}$, Fumito Kitatani ${ }^{2}$, \\ Mitsuo Koizumi ${ }^{2}$, and Harufumi Tsuchiya ${ }^{2}$ \\ 1 Standards for Nuclear Safety, Security and Safegurads Unit, JRC-Geel, EC, Retieseweg 111, 2440 Geel, Belgium \\ 2 Japan Atomic Energy Agency, Tokai-mura, Naka-gu, Ibaraki 319-1195, Japan
}

\begin{abstract}
Neutron-induced reactions can be used to study the properties of nuclear materials of interest in the fields of nuclear safeguards and security. The elemental and isotopic composition of these materials can be determined by using the presence of resonance structures. This idea is the basis of two non-destructive analysis techniques which have been developed at the GELINA neutron time-of-flight facility at JRC-Geel: Neutron Resonance Capture Analysis (NRCA) and Neutron Resonance Transmission Analysis (NRTA). A combination of NRTA and NRCA has been proposed for the characterisation of particle-like debris of melted fuel formed in severe nuclear accidents. In this work, we present a quantitative validation of the NRTA technique which was used to determine the areal densities of Pu enriched reference samples used for safeguards applications. Less than $2 \%$ bias has been obtained for the fissile isotopes, with well-known total cross sections.
\end{abstract}

\section{Introduction}

The presence of resonance structures in neutron-induced reaction cross sections is the basis of Neutron Resonance Transmission Analysis (NRTA) and Neutron Resonance Capture Analysis (NRCA) [1]. NRTA and NRCA are Non-Destructive Analysis (NDA) methods to determine the elemental and isotopic composition of materials and objects which do not require any sample preparation. They are based on the well-known methodologies used for the measurement of neutron-induced cross section performed in Time-Of-Flight (TOF) neutron facilities [2].

In particular, NRTA has been proposed as a method to quantify ${ }^{235} \mathrm{U}$ and the $\mathrm{Pu}$ isotopes in special nuclear materials (SNM), for instance the melted fuel debris formed in severe nuclear accidents [3], such as the one that occurred at the Fukushima Daiichi nuclear power plants. NRTA is an absolute NDA method that does not require any additional calibration measurement with reference samples representative for the material under investigation [1]. The accuracy of the results relies on the quality of the nuclear data, in particular, the total neutron cross sections of the nuclides present in the sample. Since total cross sections for neutron-induced reactions are one of the most accurate nuclear data, NRTA can be considered as one of the most accurate absolute NDA methods for material characterisation.

NRTA potential for the characterisation of fresh and spent nuclear fuel has already been proven by Priesmeyer and Harz [4] and by Behrens et al. [5]. Furthermore, NRTA has been applied by Noguere et al. [6] at the GELINA TOF-facility [7] to characterise a solution sample obtained from nuclear waste reprocess. However, the samples used

a e-mail: carlos.PARADELA-DOBARRO@ec . europa.eu in Refs. [4-6] were homogeneous samples with a regular shape. An analysis of particle- and powder-like debris samples of melted fuel will be much more complex and challenging [8], due to their characteristics, in particular:

- the diversity in shape and size of the particle- and powder-like debris samples,

- the presence of neutron absorbing matrix material without low energy resonances such as the boron from the control rods,

- the complexity of the transmission spectra due to the presence of resonances from the fission products, and

- the high temperature and radioactivity of the debris samples.

Most of these problems have been studied and solved as part of a collaborative effort of the Japan Atomic Energy Agency (JAEA) and the Joint Research Center (JRC) at Geel. The impact of the diversity in shape and size of the samples was investigated by means of simulations by Becker et al. [9]. Various analytical methods were studied in terms of their capability to take into account the powder characteristics and the best results were obtained using an empirical model proposed by Kopecky et al. [10] and the Levermore-Pomraning model [11]. Both models were implemented in the resonance shape analysis code REFIT [12] and validated with transmission experiments at GELINA [13]. Then, a method to account for the contribution of neutron absorbing materials without resonances in the low energy region was proposed and validated in Ref. [1] using a $\mathrm{U}_{3} \mathrm{O}_{8}$ reference sample. From measurements at the $25 \mathrm{~m}$ station of GELINA, the areal density of ${ }^{235} \mathrm{U}$ and ${ }^{238} \mathrm{U}$ was reproduced within $1 \%$ by the NRTA results despite the $99 \%$ neutron absorption in the matrix materials of the sample. 


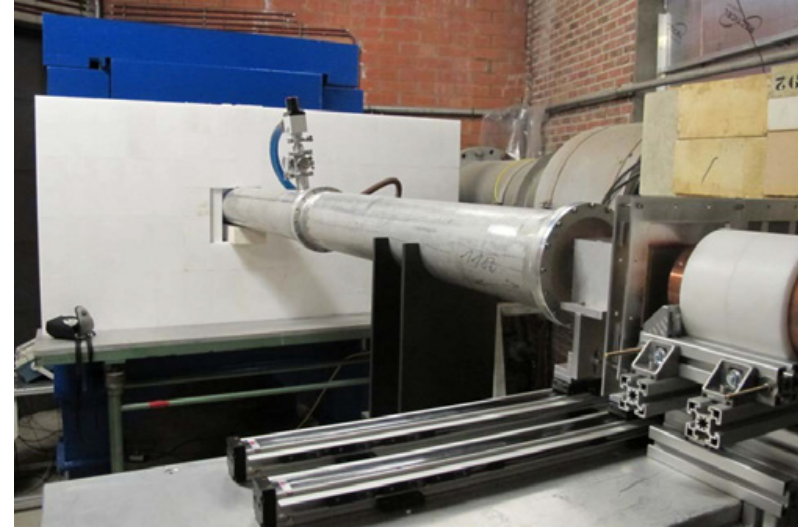

Figure 1. Picture of the transmission station at FP13 of GELINA.

All these studies and validation experiments were based on high resolution measurements performed at GELINA transmission stations with long flight paths, because, at low neutron energies, energy resolution is inversely proportional to the distance [1]. However, in order to exploit NRTA for industrial applications a more compact system with a short flight path is required. In order to investigate the performance of NRTA under such conditions and to define the requirements for an industrial system, an experimental programme was carried out at a new transmission station of GELINA. In this work we will summarise the characteristics of this new facility and the results obtained in it for the characterisation of $\mathrm{PuO}_{2}$ reference samples by means of NRTA.

\section{Compact GELINA transmission station}

In order to validate NRTA in a short flight path, a new transmission station with a nominal flight path of $10 \mathrm{~m}$ has been setup and commissioned at the flight path 13 of GELINA [14,15]. A picture of the station is shown in Fig. 1. An automatic sample changer is positioned at about $7.7 \mathrm{~m}$ from the neutron producing target, allowing an automated sequence of sample-in and sample-out measurements. Just in front of it, a second sample changer is used to mount anti-overlap filters. Neutron passing through the sample and the filters are further collimated and detected by a $6.35 \mathrm{~mm} \times 76 \mathrm{~mm} \times 76 \mathrm{~mm} \mathrm{Li}$-glass scintillator, which is placed at $11 \mathrm{~m}$ distance from the neutron target.

To assure good transmission geometry, i.e. to guarantee that all neutrons reaching the detector have passed through the sample and that neutrons which are scattered by the sample are not detected, a great effort has been carried out defining and aligning the collimation system. A set of lead, copper and nickel annular collimators with decreasing diameter were installed between the neutron producing target and the sample changer, reducing the neutron beam size to about $10 \mathrm{~mm}$ diameter at the sample position. Additional lithium and $\mathrm{B}_{4} \mathrm{C}$ collimators were installed to absorb neutrons that are scattered by the collimators. Similarly collimators with increasing diameter were positioned between the sample changer and the neutron detector to prevent scattered neutrons from reaching the detector. The collimation is modular and can be adapted to the sample size. To further minimize the

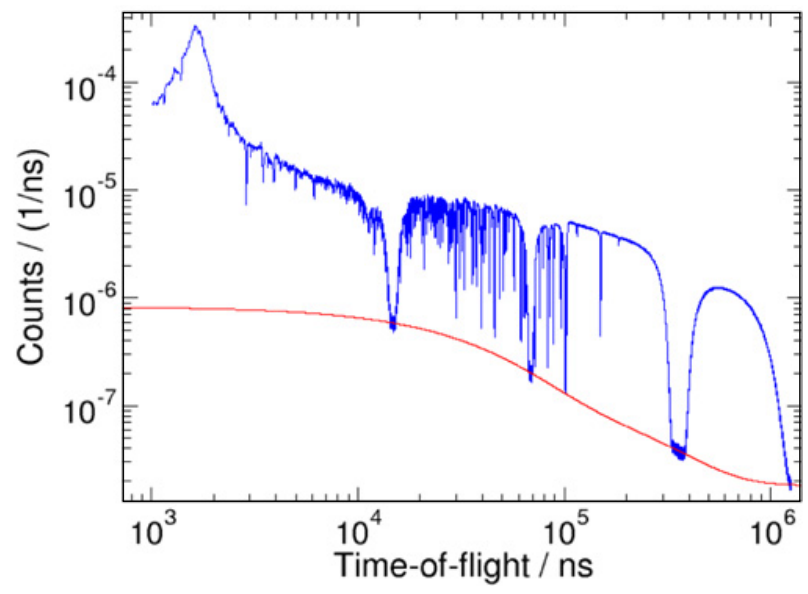

Figure 2. Transmission spectrum through $\mathrm{Na}$, $\mathrm{Co}$ and $\mathrm{Au}$ black resonance filters and $\mathrm{Cd}$ overlap filter. The red curve represents the estimated background obtained from the fit of the black resonance points.

background from scattered neutrons including those from neighbouring beam lines, the detector was placed inside a shielding structure composed of lead, boron-oxide, wax and borated polyethylene.

The transmission station was commissioned in 2014. The commissioning included an evaluation of the background conditions using two different overlap filters ( $4 \mathrm{~mm} \mathrm{Cd}$ or $12 \mathrm{~mm}$ of $\mathrm{B}$ and $\mathrm{Al}$ ) and various configurations of black resonance filters. The transmission dips due to the presence of these black resonance filters were used to approximate the background contribution by an analytical expression, as described in Ref. [2]. Results of the background characterisation are shown in Fig. 2. The TOF-spectra without sample in the beam are shown and compared with the total background. These figures reveal that a background level of less than $10 \%$ can be reached. A detailed description of the various background components in TOF transmission measurements can be found in Refs. [1,2,16].

Another important characteristic of a transmission station for NRTA applications is the TOF-response function. The response function $\mathrm{R}(t, E)$ expresses the probability that a neutron with energy $\mathrm{E}$ is observed with a time-of-flight $t$. For TOF measurements at a moderated neutron beam one of the main components determining the broadening of the observed resonance profile is the neutron transport in the target-moderator assembly. This component is best expressed in terms of an equivalent distance, as discussed in detail in Refs. [1,2]. The equivalent distance is defined as $L_{t}=v t_{t}$, where $t_{t}$ is the time difference between the moment the neutron leaves the target-moderator assembly and its time of creation. Response functions $\mathrm{R}\left(L_{t}, E\right)$, expressed as a function of $L_{t}$, do not strongly depend on the neutron energy. For flight path 13 the Full Width Half Maximum (FWHM) of the distributions is about $3.5 \mathrm{~cm}$.

\section{Characterisation of $\mathrm{PuO}_{2}$ reference materials}

A full quantitative validation of absolute NRTA results in a short flight path is obtained from measurements with a set of four $\mathrm{PuO}_{2}(\mathrm{CBNM}$ NRM 271) reference samples in this 


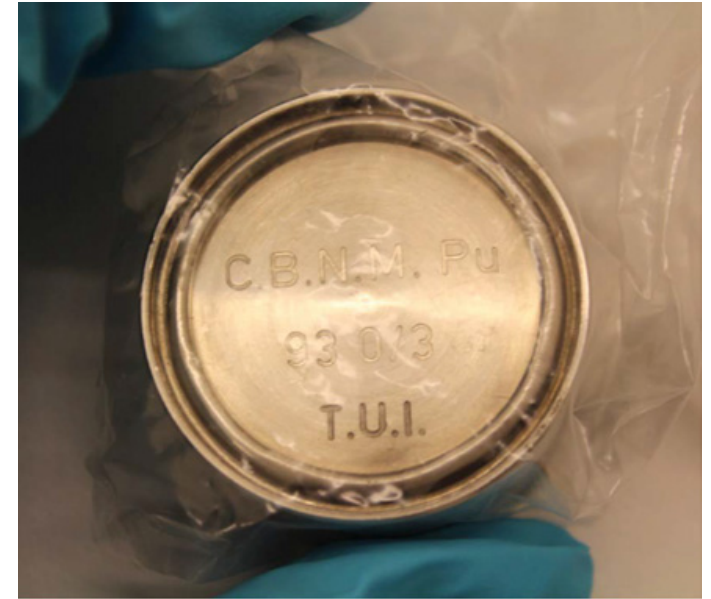

Figure 3. Picture of the $\mathrm{PuO} 2$ reference sample enriched to $93 \%$ in ${ }^{239} \mathrm{Pu}$.

new $10 \mathrm{~m}$ transmission station at GELINA. These samples were produced at JRC Geel for safeguards applications, so that they are well characterised by using different spectrometric and analytical techniques. They were sealed in stainless steel cans of $40 \mathrm{~mm}$ diameter and $21 \mathrm{~mm}$ height (see Fig. 3). The reference materials presented different enrichment in ${ }^{239} \mathrm{Pu}: 93,84,73$ and $61 \%$. In addition to the plutonium isotopes, it is worth to notice the presence of ${ }^{241} \mathrm{Am}$ which is produced by the decay of the ${ }^{241} \mathrm{Pu}$ in the sample.

Experimental transmission for each sample was obtained dividing the spectra measured with and without the sample in the beam after background subtraction. Samplein and sample-out spectra were measured alternating them in cycles of about ten minutes. The background was determined by using the black resonance technique [1], including materials with a strong resonance which removes all the neutrons of a certain energy from the beam. A Na filter was permanently present in order to determine the background level while additional measurements included $\mathrm{Co}$ and $\mathrm{W}$ filters to characterise the background energy dependence. Furthermore, measurements were performed with each one of the available overlap filters, $\mathrm{Cd}$ and ${ }^{10} \mathrm{~B}$, that remove most of the in-beam neutrons arriving after the beginning of next pulse.

An example of a transmission through the $\mathrm{PuO}_{2}$ reference sample that is enriched to $63 \%$ in ${ }^{239} \mathrm{Pu}$ is shown in Fig. 4. The result of the resonance shape analysis with REFIT to determine the areal density of the $\mathrm{Pu}$ isotopes and ${ }^{241} \mathrm{Am}$ is also shown. The resonance parameters were taken from the JEFF-3.2 data library. A comparison of the areal densities determined by NRTA and the declared values is reported in Table 1. Most of the deviations between the values determined by NRTA and the declared ones are within the uncertainties due to counting statistics. These results demonstrate that the amount of nuclear material can accurately be determined by NRTA. It should be noted that the areal densities were derived without any additional adjustment of the nuclear data. Hence, systematic deviations between declared and NRTA values might be due to limitations in the nuclear data.

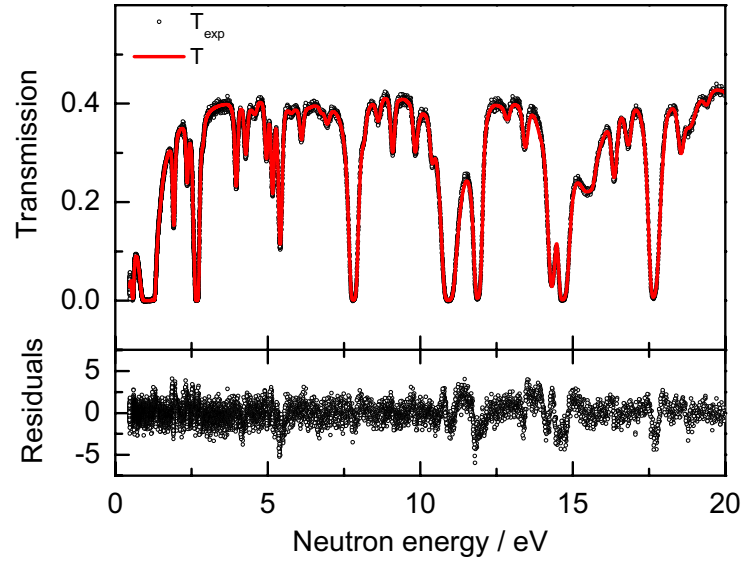

Figure 4. Transmission through the $\mathrm{PuO}_{2}$ reference sample enriched to $63 \%$ in $239 \mathrm{Pu}$. The experimental (black points) and the theoretical transmission obtained from resonance analysis (red curve) are compared using the residuals.

Table 1. Relative abundances of the Pu isotopes and ${ }^{241} \mathrm{Am}$ in the sample enriched to $63 \%$ compared with the declared values. The uncertainties on the NRTA results are only due to counting statistics.

\begin{tabular}{|l|c|c|c|}
\hline Nuclide & $\mathrm{n}_{\text {NRTA }}$ & $\mathrm{n}_{\text {REF }}$ & $\mathrm{n}_{\text {NRTA }} / \mathrm{n}_{\text {REF }}$ \\
\hline${ }^{238} \mathrm{Pu}$ & $0.0098 \pm 0.0002$ & 0.00952 & $1.029 \pm 0.020$ \\
\hline${ }^{239} \mathrm{Pu}$ & $0.625 \pm 0.001$ & 0.62602 & $0.999 \pm 0.002$ \\
\hline${ }^{240} \mathrm{Pu}$ & $0.2625 \pm 0.0002$ & 0.25272 & $1.039 \pm 0.001$ \\
\hline${ }^{241} \mathrm{Pu}$ & $0.0157 \pm 0.0001$ & 0.01564 & $1.007 \pm 0.006$ \\
\hline${ }^{242} \mathrm{Pu}$ & $0.0398 \pm 0.0001$ & 0.04149 & $0.960 \pm 0.002$ \\
\hline${ }^{241} \mathrm{Am}$ & $0.0632 \pm 0.0001$ & 0.0629 & $1.005 \pm 0.001$ \\
\hline
\end{tabular}

\section{Conclusions}

Neutron resonance transmission analysis (NRTA) was presented as a non-destructive analysis tool for the characterisation of nuclear materials. The relative abundances of the plutonium isotopes in four reference samples were determined in a $10 \mathrm{~m}$ flight path measurement at GELINA within few percent, validating the method at short flight paths.

This result opens the possibility of applying the NRTA in the nuclear safeguards and security fields or in the characterisation of spent fuel nuclear material by using compact facilities.

The authors would like to thank David Vanleeuw for providing the sample pictures.

\section{References}

[1] P. Schillebeeckx, B. Becker, H. Harada, S. Kopecky, JRC Science and Policy Reports, EUR 26848 (2014)

[2] P. Schillebeeckx, B. Becker, Y. Danon, K. Guber, H. Harada, J. Heyse, A.R. Junghans, S. Kopecky, C. Massimi, M.C. Moxon, N. Otuka, I. Sirakov, K. Volev, Nuclear Data Sheets 113, 3054 (2012)

[3] H. Harada, F. Kitatani, M. Koizumi, H. Tsuchiya, J. Takamine, M. Kureta, H. Iimura, M. Seya, B. Becker, S. Kopecky, P. Schillebeeckx, Proc. of the $35^{\text {th }}$ ESARDA Symposium on Safeguards and Nuclear Non-proliferation (Brugge, 2013)

[4] H.G. Priesmeyer, U. Harz, Atomoenergy 25, 109 (1975) 
[5] J.W. Behrens, R.A. Schrack, A.D. Carlson, C.D. Bowman, Proc. Conf. Nuclear Cross Sections for Technology (Knoxville, 1980), p. 436

[6] G. Noguere, F. Cserpak, C. Ingelbrecht, A.J.M. Plompen, C.R. Quetel, P. Schillebeeckx, Nucl. Instr. Meth. A 575, 476 (2007)

[7] W. Mondelaers, P. Schillebeeckx, Notiziario Neutroni e Luce di Sincrotrone 11, 19 (2006)

[8] P. Schillebeeckx, S. Abousahl, B. Becker, A. Borella, H. Harada, K. Kauwenberghs, F. Kitatani, M. Koizumi, S. Kopecky, A. Moens, G. Sibbens, H. Tsuchiya, ESARDA Bulletin 50, 9 (2013)

[9] B. Becker, H. Harada, K. Kauwemberghs, F. Kitatani, M. Koizumi, S. Kopecky, A. Moens, P. Schillebeeckx, G. Sibbens, H. Tsuchiya, ESARDA Bulletin 50, 2 (2013)

[10] S. Kopecky, P. Siegler, A. Moens, Proc. Int. Conf. Nuclear Data for Science and Techn. (Nice 2007), p. 623
[11] C.D. Levermore, G.C. Pomraning, D.L. Sanzo, J. Wong, J. of Math. Phys. 27, 2526 (1986)

[12] M.C. Moxon, J.B. Brisland, Technical Report AEAINTEC-0630 (AEA Technology, 1991)

[13] B. Becker, S. Kopecky, H. Harada, P. Schillebeeckx, Eur. Phys. J. Plus 129, 58 (2014)

[14] J. Heyse, B. Becker, H. Harada, M. Koizumi, S. Kopecky, C. Paradela, P. Schillebeeckx, H. Tsuchiya, Proc. of $14^{\text {th }}$ Int. Conf. on Advancements in Nuclear Instrumentation Measurement Methods and their Applications ANIMMA (Lisbon, 2015)

[15] C. Paradela, G. Alaerts, B. Becker, J. Heyse, S. Kopecky, A. Moens, W. Mondelaers, P. Schillebeeckx, R. Wynants, H. Harada, F. Kitatani, M. Koizumi, H. Tsuchiya, Il Nuovo Cimento 38C, 176 (2015)

[16] I. Sirakov, B. Becker, R. Capote, E. Dupont, S. Kopecky, C. Massimi, P. Schillebeeckx, Eur. Phys. J. A 49, 144 (2013) 